\title{
ZONEAMENTO AGRO-CLIMÁTICO DA CANA-DE-AÇÚCAR PARA O ESTADO DE MINAS GERAIS COM BASE EM REGRESSÕES MÚLTIPLAS
}

\author{
THOMÉ SIMPLICIANO ALMEIDA ${ }^{1}$, MARIA LÚCIA CALIJURI ${ }^{2}$, LUCIANA BARROS PINTO \\ ${ }^{1}$ Instituto Federal do Sul de Minas (IFsuldeminas), Passos, MG, Brasil \\ ${ }^{2}$ Universidade Federal de Viçosa (UFV), Viçosa, MG, Brasil \\ ${ }^{3}$ Universidade Federal de Pelotas (UFPEL), Pelotas, RS, Brasil
}

thome.almeida@ifsuldeminas.edu.br, calijuri@ufv.br, luciana.pinto@ufpel.edu.br

Recebido Novembro de 2010 - Aceito Março de 2013

\begin{abstract}
RESUMO
O objetivo deste trabalho foi identificar as regiões aptas, restritas e inaptas ao cultivo da cana-deaçúcar no estado de Minas Gerais, através da geoespacialização e regressão múltipla. Utilizaram-se dados de temperatura e déficit hídrico de 32 estações meteorológicas instaladas no estado. Os dados de déficit hídrico foram determinados utilizando o balanço hídrico segundo Thornthwaite e Mather (1955). A maior parte do estado é restrita ao cultivo da cultura, conforme os critérios de produtividade relacionados com as exigências térmicas e hídricas da cultura.
\end{abstract}

Palavras-chave: aptidão climática, temperatura e déficit hídrico.

\begin{abstract}
AGRO-CLIMATOLOGICAL ZONING OF CANE SUGAR FOR THE MINAS GERAIS STATE BASED ON MULTIPLE REGRESSIONS

It was aimed in this work, through the geospatialization and multiple regression analysis, the identification of the suitable, restricted and unsuitable regions for cultivation of sugar cane in Minas Gerais State. It was used temperature and water deficit data from 32 meteorological stations located in the State. The data of water deficit were determined using the water balance according to Thornthwaite and Mather (1955) method. Most of the state is restricted to the cultivation, according to the productivity criteria related with thermal and hydric demands of the culture.
\end{abstract}

Keywords: climate suitability, temperature and water deficit.

\section{INTRODUÇÃO}

Atualmente a demanda pela produção de fontes de energia renováveis e a preocupação com a preservação do meio ambiente tem cobrado de todos os países do mundo uma atitude com relação a tal fato. O Brasil, nos últimos anos, está em evidência pela sua potencialidade de exploração agrícola, e dos diversos tipos de alimentos e fontes de energia.

Com relação às culturas bioenergéticas, alto destaque é dado à cana-de-açúcar no Brasil, onde em 2008 ocorreu um recorde de produção de acordo com o levantamento feito pela Companhia Nacional de Abastecimento (CONAB).

$\mathrm{O}$ estabelecimento de políticas que norteiem o desenvolvimento da agricultura sucroalcooleira é necessário, no sentido de garantir a produção de cana-de-açúcar para suprir a demanda de combustível e prover adequada produção de alimento.
Atualmente, o Brasil é o maior produtor mundial de cana de açúcar, com mais de 8,2 milhões de hectares, chegando a $40 \%$ do total do comércio mundial em 2006 (Colin, 2009). Em 2008 a produtividade foi de 654,3 milhões de toneladas, com produtividade de 79,27 t/ha (IBGE, 2010).

O estado de Minas Gerais é o segundo maior produtor do país, com grande expansão na última década, tendo participação em $8,75 \%$ da produção nacional, com uma produção acima de 61 milhões de toneladas (IBGE, 2010). Em destaque, tem-se a região do Triângulo Mineiro, com os municípios de Uberaba, Frutal, Ituiutaba, Conceição das Alagoas e Iturama.

Nesse sentido, o zoneamento agrícola é fundamental para organização dos programas de trabalho e suporte do planejamento da agricultura. Este é baseado em fatores que definem as aptidões agrícolas, as quais diferem de região para região. O conhecimento das condições climáticas de determinada região é de extrema importância, pois uma vez conhecidas às 
regiões climaticamente homogêneas, pode-se estabelecer os indicadores do meio físico e biológico dessa região que são compatíveis ou não com a exploração de determinadas culturas (Nunes et al., 2007).

Um zoneamento agro-climático para a cana-de-açúcar no estado de Minas Gerais foi realizado na década 60 (GEOMINAS, 2009), fazendo a interpolação dos dados necessários para indicar as áreas aptas, restritas e inaptas ao cultivo da cultura. Porém, o zoneamento agrícola de uma região deve ser constantemente atualizado visando obter maiores informações sobre as condições climáticas das culturas selecionadas e, sobretudo, proporcionar maior retorno dos investimentos a médio e longo prazo para os produtores (Rossetti, 2001). Há necessidade, portanto, da organização de banco de dados mais completos e consistentes, bem como, a utilização de técnicas mais modernas e sofisticadas no delineamento dos limites climáticos para o atendimento à adaptabilidade de novas variedades (Sediyama et al., 2001).

Devido à carência de dados climatológicos, principalmente quando se trata de grandes áreas, os métodos para o zoneamento agro-climático têm explorado a interpolação espacial dos dados climáticos, onde se despreza a interferência de fatores que influenciam no clima da região como relevo, latitude e longitude. Os mapas de regionalização das informações de alguns elementos climáticos, sendo conhecidas as equações que representam as normais anuais, possibilitam a análise da abrangência geográfica dos mesmos, materializando os valores desses elementos em forma de mapas georreferenciados (Sediyama e Melo, 1998).

Os principais componentes climáticos que controlam o crescimento, a produção e qualidade da cana são temperatura e umidade do ar. $\mathrm{O}$ crescimento da cana está intimamente ligado à temperatura, tendo relação com o nível de sacarose e até inibindo a brotação (no caso de temperatura do ar muito baixa). A deficiência hídrica do solo afeta a produtividade da cana, que quando não satisfatória, pode acarretar em um baixo desenvolvimento das raízes e baixo perfilhamento, resultando, portanto num baixo aproveitamento da água e nutrientes disponíveis (Rojas e Barbieri, 1999). Leite et al. (2009) afirmam que o clima do país permite o cultivo de cana-de-açúcar a altos rendimentos com pouca ou nenhuma irrigação.
Portanto, este trabalho tem por objetivo propor um zoneamento agroclimático para o plantio de cana-de-açúcar no estado de Minas Gerais, baseado na espacialização de dados de temperatura normal climatológica e déficit hídrico, relacionando a espacialização com relevo, latitude e longitude, utilizando equações de regressão e sistema de informação geográfica.

\section{MATERIAIS E MÉTODOS}

A área de estudo abrangeu o estado de Minas Gerais e utilizaram-se dados do Instituto Nacional de Meteorologia (INMET) de 32 estações meteorológicas no estado. Essas estações continham mais que 30 anos de dados, sendo assim possível o cálculo das normais climatológicas. Com esses dados, calculou-se o balanço hídrico segundo Thornthwaite, modificado por Mather (Thornthwaite e Mather, 1955) obtendo-se, assim, a deficiência hídrica anual (Da) para cada localidade.

As análises de regressão foram efetuadas com os dados das temperaturas médias normais anuais do ar e deficiência hídrica anual do solo, segundo o seguinte modelo:

$$
\begin{aligned}
& Y_{i}=a_{0}+a_{1} x_{1}+a_{2} x_{2}+a_{3} x_{3}+e_{1} \\
& Y_{j}=b_{0}+b_{1} x_{1}+b_{2} x_{2}+b_{3} x_{3}+e_{2}
\end{aligned}
$$

em que:

$\mathrm{Y}_{\mathrm{i}}=$ temperatura média normal anual estimada;

$\mathrm{Y}_{\mathrm{j}}=$ deficiência hídrica anual estimada;

$\mathrm{x}_{1}=$ latitude do local em graus e décimos;

$\mathrm{x}_{2}=$ longitude do local em graus e décimos;

$\mathrm{x}_{3}=$ altitude do local em metros;

$\mathrm{e}=$ erro.

De posse das equações de regressão foram utilizados os mapas de altitude (SRTM - Shuttle Radar Topography Mission), latitude e longitude (dos pontos do estado) para a estimativa da temperatura média anual e deficiência hídrica anual para todo o estado.

Os índices térmicos exigidos pela cultura e seu desenvolvimento foram classificados de acordo com as necessidades das culturas, dividindo-se em três níveis: apto, restrito e inapto, como segue:

Tabela 1 - Exigências climáticas anuais da cana-de-açúcar.

\begin{tabular}{ccc} 
Apta & Restrita & Inapta \\
\hline $40 \mathrm{~mm}<\mathrm{Da}<300 \mathrm{~mm}$ & $\mathrm{Da}>300 \mathrm{~mm}$ & $\mathrm{Da}<40 \mathrm{~mm}$ \\
$\mathrm{e}$ & ou & ou \\
$22^{\circ} \mathrm{C}<\mathrm{T}<38^{\circ} \mathrm{C}$ & $18^{\circ} \mathrm{C}<\mathrm{T}<22^{\circ} \mathrm{C}$ & $\mathrm{T}<18^{\circ} \mathrm{C}$
\end{tabular}

Fonte: Embrapa (2004). 
- Apto: As condições térmicas e hídricas da área apresentam-se favoráveis para o bom desenvolvimento e produção da cultura em escala econômica.

- Restrito: Apresentam condições restritas quanto ao regime hídrico ou térmico, ou ambos, que podem eventualmente prejudicar as fases de desenvolvimento da cultura, repercutindo negativamente na produção.

- Inapto: As características normais de clima são inadequadas à exploração econômica da cultura, por apresentar limitações severas dos fatores hídricos, térmicos ou ambos.

A partir dos mapas de temperatura e deficiência hídrica, procedeu-se uma classificação de áreas de acordo com as exigências climáticas das culturas e sua aptidão (Tabela 1).

\section{RESULTADOS E DISCUSSÃO}

As Equações 3 e 4 apresentam as equações de regressão múltipla e os respectivos coeficientes de determinação, conforme os modelos propostos nas Equações 1 e 2 .

$$
\mathrm{Yi}=24,81-0,5039 \mathrm{x}_{1}+0,2248 \mathrm{x}_{2}-0,00537 \mathrm{x}_{3}
$$

com $R^{2}=0,93$.

$$
Y_{j}=1114,5816+43,6337 x_{1}+0,02341 x_{2}-0,10448 x_{3}
$$

$\operatorname{com} \mathrm{R}^{2}=0,78$.

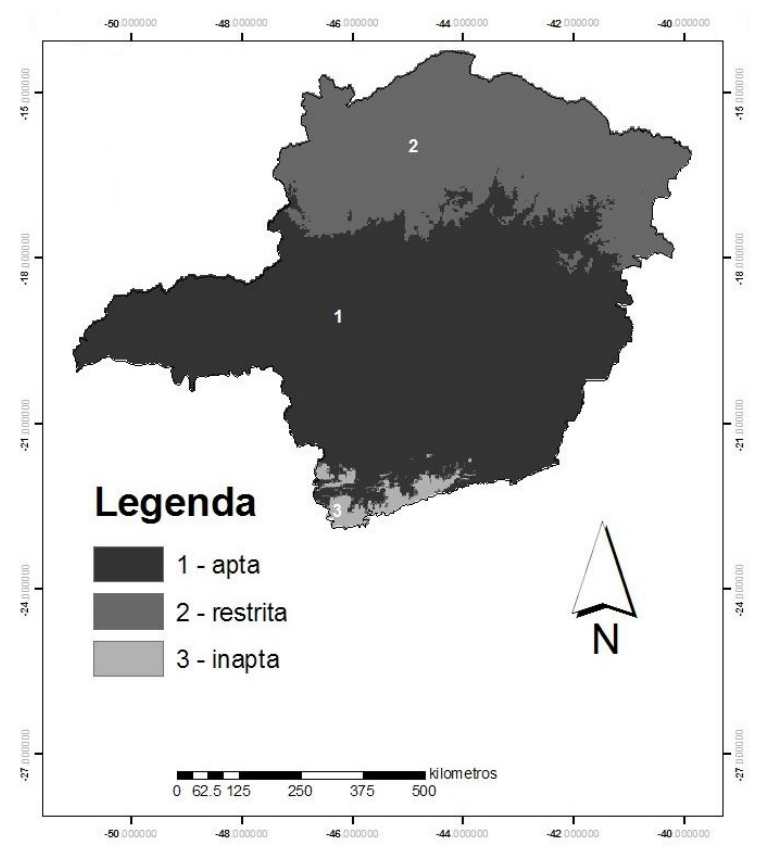

Figura 1 - Aptidão hídrica da cana-de-açúcar para o estado de Minas Gerais.
As duas equações de regressão foram significativas ao nível de 5\% de probabilidade, pelo teste de $\mathrm{F}$.

Assim, foram construídos os mapas de temperatura média anual e deficiência hídrica anual com as Equações 3 e 4 , através da álgebra dos mapas de altitude, latitude e longitude do estado, aplicando as regressões para cada ponto do estado.

Os resultados referentes às zonas de aptidão ao déficit hídrico para a cultura de cana no estado de Minas Gerais são apresentados na Figura 1. No mapa de aptidão ao déficit hídrico, é possível observar que, mais da metade do estado possui condições favoráveis ao cultivo do café, abrangendo, em maioria, a região central do estado. À medida que se aproxima da região norte do estado, observam-se restrições ao cultivo da cana, em razão dos menores índices de precipitação pluvial, e assim maior demanda evapotranspirativa, fatores que, de modo geral, condicionam baixo armazenamento de água no solo restringindo, portanto, o cultivo da cana.

Com relação à aptidão térmica (Figura 2), observa-se que as maiores áreas que contém condições favoráveis ao plantio da cana estão em uma faixa que se estende da região do Triângulo Mineiro e parte do nordeste e norte de Minas Gerais. As regiões centrais e algumas áreas isoladas do estado apresentam condições térmicas restritas ao cultivo de cana, enquanto que pequenas áreas situadas na região sul do estado apresentam inaptidão térmica, sendo locais impróprios para o cultivo.

A Figura 3 apresenta o zoneamento climático da aptidão ao cultivo da cana-de-açúcar para o estado de Minas Gerais, onde os resultados mostram que, sob o ponto de vista climático,

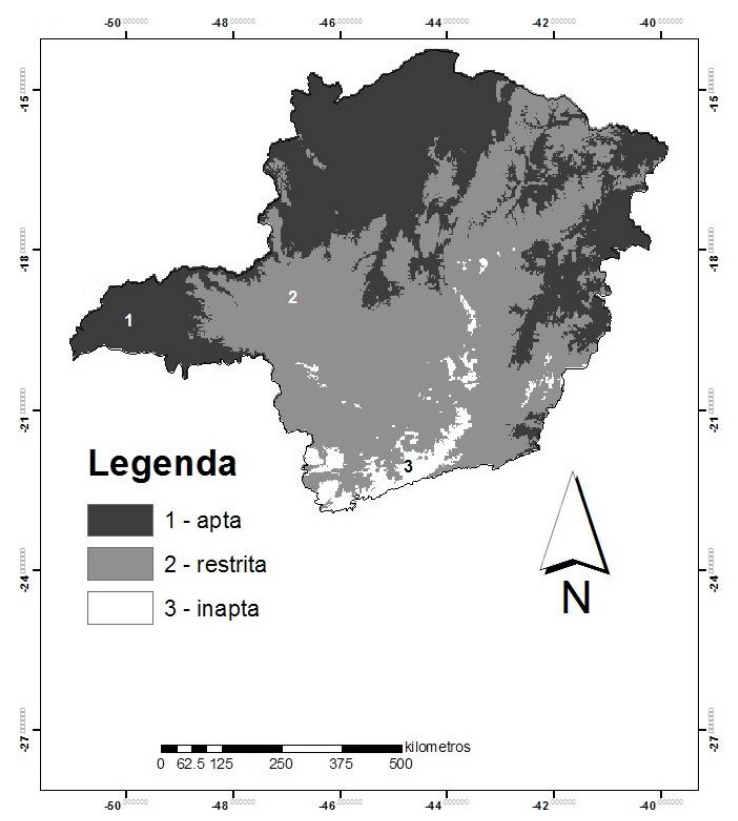

Figura 2 - Aptidão térmica da cana-de-açúcar para o estado de Minas Gerais. 


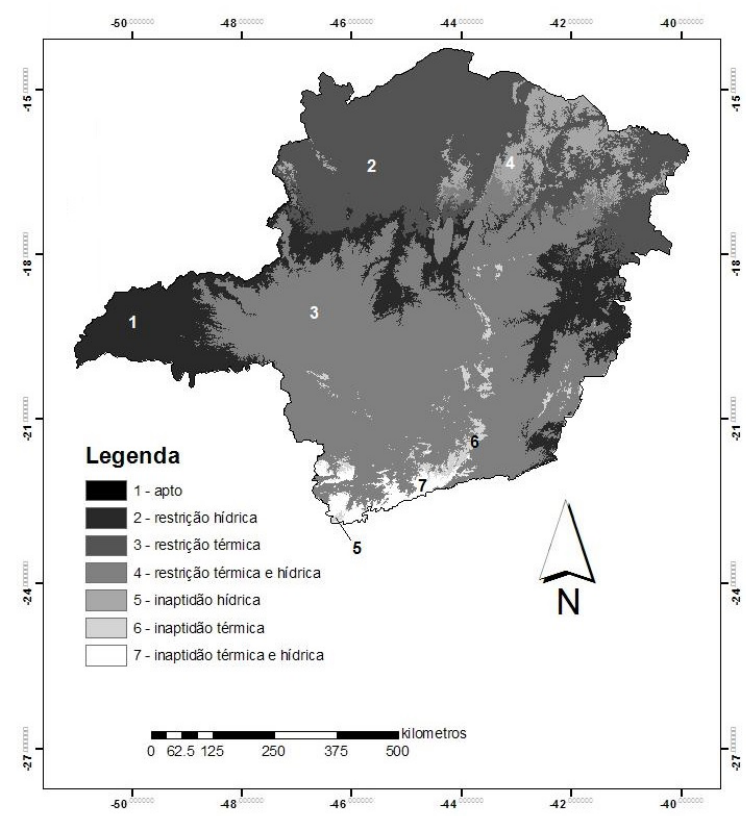

Figura 3 - Aptidão agro-climática para a cana-de-açúcar no estado de Minas Gerais.

somente parte do Triângulo Mineiro e algumas áreas isoladas são aptas ao cultivo da cana. Na maior área do estado a cana pode ser cultivada, embora apresentando alguma restrição devido à temperatura do ar ou deficiência hídrica. Nessas regiões o cultivo pode ser realizado adotando-se técnicas de manejo, como por exemplo, a suplementação de água por irrigação, de modo a ajustar o fator restritivo e tornar essas áreas potencialmente produtivas.

Em comparação com o zoneamento agro-climático de 1960 (Geominas), observa-se uma redução das áreas aptas principalmente na região central do estado, que se apresentaram agora como restrita.

\section{CONCLUSÕES}

1. O zoneamento agro-climático da cultura de canade-açúcar indicou as áreas aptas, restritas e inaptas ao cultivo, sendo que no Triângulo Mineiro apresenta a maior área apta climatologicamente.

2. As equações de regressão, para a estimativa da temperatura média anual e deficiência hídrica anual, tiveram bons valores de coeficiente de determinação e foram significativas ao nível de 5\%, pelo teste de $\mathrm{F}$.

3. Em comparação com o zoneamento feito na década de 60, observa-se uma redução das áreas aptas ao cultivo da cana, principalmente na área central do estado.

\section{REFERÊNCIAS BIBLIOGRÁFICAS}

Colin, E. C. Mathematical programming accelerates implementation f agro-industrial sugarcane complex. European Journal of Operational Research, v 199, p.232-235, 2009.

Empresa Brasileira de Pesquisa Agropecuária (Embrapa). 2004. Sistemas de Produção. Disponível em: http:// sistemasdeproducao.cnptia.embrapa.br. 15 Abril. 2009.

GEOMINAS. Zoneamento Agroclimático de Minas Gerais Aptidões Agrícolas 1996 Cultura de Cana-de-açúcar. http://www.geominas.mg.gov.br. 01 Maio. 2009.

IBGE - Sistema de Recuperação Automática - SIDRA. 2010. Disponível em: hattp://www.sidra. ibge.gov.br. Acesso em 19 de dezembro de 2011.

Leite, R. C. C.; Leal, M. R. L. V.; Cortez, L. S. B.; Griffin, W. M.; Scandiffio, M. I. G. Can Brazil replace $5 \%$ of the 2025 gasoline world demand with ethanol? Energy, v.34, n.5, p.655-661, 2009.

Nunes, E. L.; Amorim, R. C. F. de; Souza, W. G. de; Ribeiro, A.; Senna, M. C. A.; Leal, B. G. Zoneamento agroclimático da cultura do café para a bacia do Rio Doce. Revista Brasileira de Meteorologia, v.22, n.3, p.297-302, 2007.

Rojas, J. S. D.; Barbieri V. Modelo agrometoorológico de estimativa da produtividade da cana-de-açucar. Revista Brasileira de Agrometeorologia, , v.7, n.1, p.67-73, 1999.

Rosseti, L.A. Zoneamento agrícola em aplicações de crédito e securidade rural no Brasil: aspectos atuariais e de política agrícola. Revista Brasileira de Agrometeorologia, v.9, n.3, (No Especial: Zoneamento Agrícola), p. 386-399, 2001.

Sediyama, G. C.; Melo, J.C. Modelos para a estimativa das temperaturas normais mensais médias, máximas, mínimas e anual no estado de Minas Gerais. Engenharia na Agricultura, v.6 n.1, p.57-61, 1998.

Sediyama, G. C. et al. Zoneamento agroclimático do cafeeiro (Coffea arábica L.) para o estado de Minas Gerais. Revista Brasileira de Agrometeorologia, v. 9, n. 3, p.501-509, 2001.

Thornthwaite, C.W.; Mather, J.R. The water balance. Laboratory of Climatology, v.8 n.1, p.1-14, 1955. 